\title{
Robust Adaptive Fuzzy Control of Uncertain Nonlinear Systems with Unknown Dead-Zone
}

\author{
Chiang-Cheng Chiang
}

Department of Electrical Engineering, Tatung University, 40 Chung Shan North Road, Sec.3, Taipei, Taiwan, Republic of China

\begin{abstract}
This paper presents a robust adaptive fuzzy control scheme for a class of uncertain nonlinear systems containing an unknown dead-zone. Dead-zone characteristics are quite commonly encountered in actuators, such as hydraulic and pneumatic valves, electric servomotors, and electronic circuits, etc. Therefore, by using a description of a dead-zone and by exploring the properties of this dead-zone model intuitively and mathematically, a robust adaptive fuzzy control method is presented without constructing the dead-zone inverse. The unknown nonlinear functions of the controlled system are approximated by the fuzzy logic system according to some adaptive laws. By means of Lyapunov stability theorem, the proposed robust adaptive fuzzy control scheme can guarantee the robust stability of the whole closed-loop system with an unknown dead-zone in the actuator and obtain good tracking performance as well. Finally, an example and simulation results are illustrated to demonstrate the validity of the proposed method.
\end{abstract}

Keywords Lyapunov Stability Theorem, Nonlinear Systems, Dead-Zone, Fuzzy Logical System, Robust Adaptive Control

\section{Introduction}

Generally, many practical control systems have the structure of a dynamical system preceded by some nonsmooth nonlinearities in the actuator, such as dead-zone, backlash, saturation, etc[1-3]. Furthermore, these nonsmooth nonlinearities in such actuators give rise to undesirable inaccuracy or oscillations or even lead to instability, whose parameters are often unknown and may vary with time. Thus, the study of dead-zone is particularly important when the expected accuracy of the dynamical system is high, and the control problem of these nonsmooth nonlinearities has attracted considerable attention of a number of researchers over the past years[4-6].

In the controller design of nonlinear systems, the feedback linearization methods have been widely studied and developed, and these methods can be utilized to transform a nonlinear dynamic system into a linear system by means of state feedback approach[7-8]. However, these methods can only be applied to nonlinear systems whose dynamics must be exactly known. If there exist uncertainties in those nonlinear terms, or the nonlinear terms are completely unknown, it is difficult to achieve the exact feedback linearization such that the nonlinear control systems have poor performance. In this study, we intend to apply adaptive

* Corresponding author:

ccchiang@ttu.edu.tw (Chiang-Cheng Chiang)

Published online at http://journal.sapub.org/ajis

Copyright (C) 2012 Scientific \& Academic Publishing. All Rights Reserved fuzzy modeling control schemes to deal with the unknown uncertain nonlinear systems preceded by an unknown dead-zone.

Fuzzy logic control has recently found extensive applications for systems that are complex and ill-defined. Based on the universal approximation theorem, several stable adaptive fuzzy control schemes[9-14] have been developed to overcome the difficulty of extracting linguistic control rules from experts and to cope with the system parameter changes. An adaptive fuzzy system is a fuzzy logic system equipped with an adaptation algorithm to be able to update the fuzzy system parameters[15-16], and the fuzzy logic system is constructed from a collection of fuzzy IF-THEN rules. According to the definition in[15], the adaptive fuzzy approaches can be classified as: direct adaptive fuzzy control and indirect adaptive fuzzy control. The direct adaptive fuzzy control scheme uses fuzzy systems to describe the control action, and the parameters of the fuzzy system are adjusted directly to satisfy the required control objective[10, 12, 19]. In contrast with the direct adaptive fuzzy control scheme, an indirect adaptive fuzzy control scheme uses fuzzy systems to estimate the plant dynamics, and a suitable controller is developed for the estimated system[9-10, 13, 17-18]. In this study, due to the nonlinearity of dead zone and uncertainties, the indirect adaptive fuzzy control scheme is adopted to tackle the control problem of these nonlinear systems.

Variable structure control (VSC) has been studied extensively and received many applications in recent years. It has been shown that variable structure control possesses 
several advantages, such as fast response, robustness of stability, insensitivity to the matching parameters variations, and external disturbances[22-24]. Sliding mode control (SMC) methods can be considered as a synthesis procedure, which is often associated with the theory of variable structure control (VSC). Sliding mode control (SMC) design technique presents simple control laws which constrain the system motion on suitably chosen manifolds. However, when the dynamics of controlled system are in the switching hyperplane, a switching function must be used in the control law, which causes chattering of the control signals. Chattering is the main drawback of SMC, which can excite undesirable high-frequency dynamics. In order to reduce chattering phenomenon, a small boundary layer[25-26] is introduced around the sliding surface for better control accuracy.

In this paper, in order to eliminate the effects arising from the dead-zone input nonlinearity, a robust adaptive fuzzy control scheme is proposed to overcome the stabilization problem of a class of uncertain nonlinear systems preceded by an unknown dead-zone. In the previous works[27-29], a common feature is the construction of an inverse dead-zone nonlinearity to minimize the effects of dead-zone. But, unlike the aforementioned method, by using a description of a dead-zone and exploring the properties of this dead-zone model intuitively and mathematically, a robust adaptive fuzzy control method is presented without constructing the dead-zone inverse. Finally, based on the Lyapunov stability theorem and the theory of variable structure control, the proposed robust adaptive fuzzy control scheme can guarantee the robust stability of the whole closed-loop system with an unknown dead-zone in the actuator and obtain good tracking performance as well.

The remainder of this paper is organized as follows. In Section 2, the problem formulation is presented, and a detailed description of fuzzy logic systems and fuzzy basis functions is reviewed. Section 3 introduces robust adaptive fuzzy modeling control scheme to deal with the robust control problem for a class of uncertain nonlinear systems with an unknown dead-zone. Moreover, the robust stability condition will be derived by means of the Lyapunov approach. The simulation results are illustrated in Section 4 to show the effectiveness of the proposed robust adaptive fuzzy controller. Finally, a conclusion is given in Section 5 .

\section{Problem Formulation and Preliminaries}

\subsection{Problem Statement}

Consider a class of the following uncertain nonlinear system with an unknown dead-zone of the form

$$
\left\{\begin{array}{l}
\dot{x}_{1}=x_{2} \\
\dot{x}_{2}=x_{3} \\
\quad \vdots \\
\dot{x}_{n}=f(\mathbf{x})+\Delta f(\mathbf{x})+g(\mathbf{x}) D(v(t)) \\
y=x_{1}
\end{array}\right.
$$

or equivalently

$$
\left\{\begin{array}{l}
x^{(n)}=f(\mathbf{x})+\Delta f(\mathbf{x})+g(\mathbf{x}) D(v(t)) \\
y=x
\end{array}\right.
$$

where $\mathbf{x}=\left[x_{1}, x_{2}, \cdots, x_{n}\right]^{T}=\left[x, \dot{x}, \cdots, x^{(n-1)}\right]^{T} \in R^{n}$ is the system state vector which is assumed to be available for measurement, $v \in R$ and $y \in R$ are the input and output of the system, respectively, $f$ and $g$ are unknown nonlinear functions and $\Delta f$ is the unknown uncertainty. Without loss of generality, it is assumed that the sign of $g(\mathbf{x})$ is positive, and $|\Delta f(\mathbf{x})| \leq h(\mathbf{x})$, where $h(\mathbf{x})$ is an unknown continuous function and can be estimated by an adaptive law in the latter. $D(v(t)): R \rightarrow R$ is the nonlinear input function containing a dead-zone.

To clarify the dead-zone nonlinear input function $D(\cdot)$, the dead-zone with input $v(t)$ and output $w(t)$, as shown in Fig. 1, is described by

$$
w(t)=D(v(t))= \begin{cases}m_{r}\left(v(t)-b_{r}\right) & \text { for } v(t) \geq b_{r}, \\ 0 & \text { for } b_{l}<v(t)<b_{r}, \\ m_{l}\left(v(t)-b_{l}\right) & \text { for } v(t) \leq b_{l},\end{cases}
$$

where $b_{r}>0, b_{l}<0$ and $m_{r}>0, m_{l}>0$ are parameters and slopes of the dead-zone, respectively. In order to investigate the key features of the dead-zone in the control problems, we have the following assumptions:

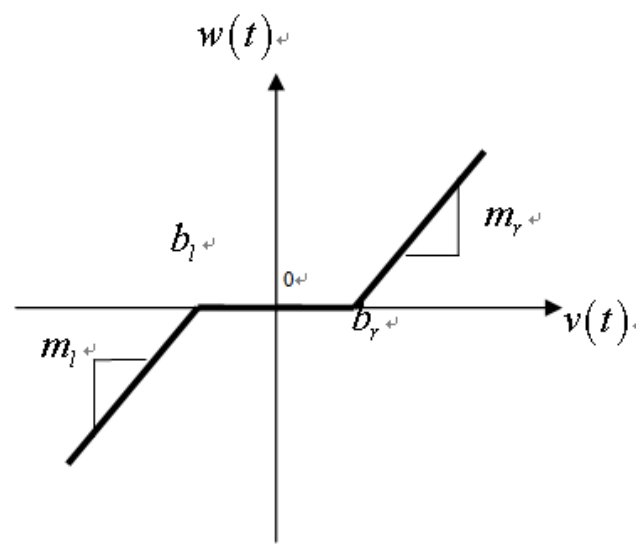

Figure 1. Dead-zone model 
Assumption 1: The dead-zone output $w(t)$ is not available.

Assumption 2: The dead-zone slopes are same, i.e. $m_{r}=m_{l}=m$

Assumption 3: There exist known constants $b_{r \text { min }}$, $b_{r \max }, b_{l \min }, b_{l \max }, m_{\min }, m_{\max }$ such that the unknown dead-zone parameters $b_{r}, b_{l}$, and $m$ are bounded, i.e. $b_{r} \in\left[b_{r \min }, b_{r \max }\right], b_{l} \in\left[b_{l \min }, b_{l \max }\right]$, and $m \in\left[m_{\min }, m_{\max }\right]$.

Based on the above assumptions, the expression (3) can be represented as

$$
w(t)=D(v(t))=m v(t)+d(v(t)),
$$

where $d(v(t))$ can be calculated from (3) and (4) as

$$
d(v(t))= \begin{cases}-m b_{r} & \text { for } v(t) \geq b_{r}, \\ -m v(t) & \text { for } b_{l}<v(t)<b_{r}, \\ -m b_{l} & \text { for } v(t) \leq b_{l} .\end{cases}
$$

From Assumptions 2 and 3, one can conclude that $d(v(t))$ is bounded, and satisfies

$$
|d(v(t))| \leq \rho,
$$

where $\rho$ is the upper-bound, which can be chosen as

$$
\rho=\max \left\{m_{\max } b_{r \max },-m_{\max } b_{l \min }\right\},
$$

where $b_{l \text { min }}$ is a negative value.

Let $y_{m}$ be a given bounded reference signal and contain finite derivatives up to the $n$th order. Define the tracking error

$$
e_{1}=y_{m}-y \text {. }
$$

and $\mathbf{y}=\left[y, \dot{y}, \cdots, y^{(n-1)}\right]^{T}$ and $\mathbf{y}_{\mathbf{m}}=\left[y_{m}, \dot{y}_{m}, \cdots, y_{m}^{(n-1)}\right]^{T}$.

The control objective of this paper is to design a control law $v(t)$ such that $y$ can follow a given desired reference signal $y_{m}$, under the constraint that all signals involved must be bounded.

\subsection{Description of Fuzzy Logic Systems}

Fig. 2 shows the basic configuration of the fuzzy logic system which consists of four main components: fuzzy rule base, fuzzy inference engine, fuzzifer and defuzzifer[15-16]. The fuzzy logic system performs a mapping from $U \subset R^{n}$ to $V \subset R$. Let $U=U_{1} \times \cdots \times U_{n}$ where $U_{i} \subset R$, $i=1,2, \cdots, n$. The fuzzifer maps a crisp point in $U$ into a fuzzy set in $U$. The fuzzy rule base consists of a collection of fuzzy IF-THEN rules:

$$
\begin{aligned}
& R^{(l)}: \text { IF } x_{1} \text { is } F_{1}^{l} \text {, and } x_{2} \text { is } F_{2}^{l} \text {, and } \cdots \text { and, } x_{n} \text { is } F_{n}^{l} \\
& \text { THEN } y \text { is } G^{l} \text {, }
\end{aligned}
$$

in which $\mathbf{x}=\left[x_{1}, x_{2}, \cdots, x_{n}\right]^{T} \in U$ and $y \in V \subset R$ are the input and output of the fuzzy logic system, $F_{i}^{l}$ and $G^{l}$ are fuzzy sets in $U_{i}$ and $V$, respectively. The fuzzy inference engine performs a mapping from fuzzy sets in $U$ to fuzzy sets in $V$, based upon the fuzzy IF-THEN rules in the fuzzy rule base and the compositional rule of inference. The defuzzifier maps a fuzzy set in $V$ to a crisp point in $V$.

The fuzzy systems with center-average defuzzifer, product inference and singleton fuzzifier are of the following form:

$$
y(\mathbf{x})=\frac{\sum_{l=1}^{M} \theta^{l}\left(\prod_{i=1}^{n} \mu_{F_{i}^{l}}\left(x_{i}\right)\right)}{\sum_{l=1}^{M}\left(\prod_{i=1}^{n} \mu_{F_{i}}\left(x_{i}\right)\right)}
$$

where $\theta^{l}$ is the point at which fuzzy membership function $\mu_{G^{l}}\left(\theta^{l}\right)$ achieves its maximum value, and we assume that $\mu_{G^{l}}\left(\theta^{l}\right)=1$. Eq. (9) can be rewritten as

$$
y(\mathbf{x})=\boldsymbol{\theta}^{T} \xi(\mathbf{x})
$$

where $\boldsymbol{\theta}=\left[\theta^{1}, \theta^{2}, \cdots, \theta^{l}\right]^{T}$ is a parameter vector, and $\xi(\mathbf{x})=\left[\xi^{1}(\mathbf{x}), \cdots, \xi^{M}(\mathbf{x})\right]^{T}$ is a regressive vector with the regressor $\xi^{l}(\mathbf{x})$, which is defined as fuzzy basis function

$$
\xi^{l}(\mathbf{x})=\frac{\prod_{i=1}^{n} \mu_{F_{i}^{l}}\left(x_{i}\right)}{\sum_{l=1}^{M}\left(\prod_{i=1}^{n} \mu_{F_{i}^{l}}\left(x_{i}\right)\right)}
$$

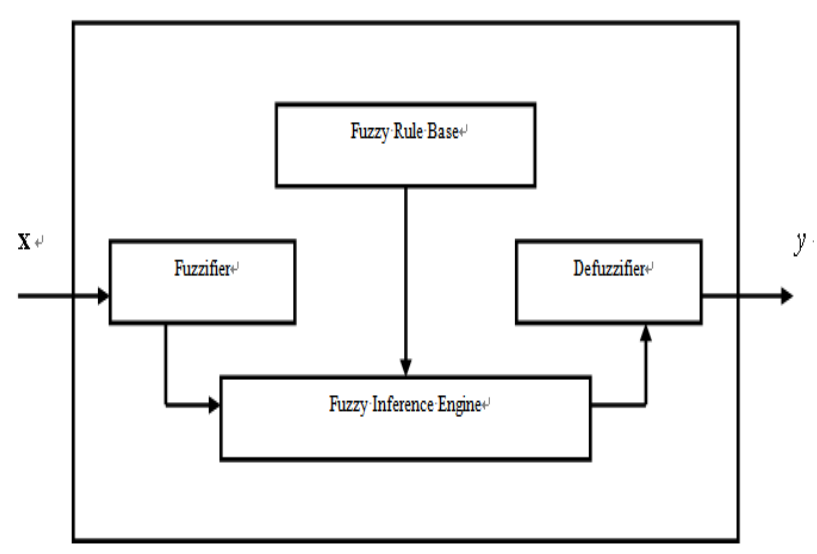

Figure 2. The basic configuration of fuzzy logic system

\section{Controller Design and Stability Analysis}


In this section, we synthesize the robust adaptive fuzzy controller to deal with the control problem of uncertain nonlinear systems with unknown dead zone.

First, let

$$
\begin{aligned}
& e_{1}=y_{m}-y=y_{m}-x_{1}, \\
& e_{2}=\dot{y}_{m}-\dot{y}=\dot{y}_{m}-x_{2}, \\
& \quad \vdots \\
& e_{n}=y_{m}^{(n-1)}-y^{(n-1)}=y_{m}^{(n-1)}-x_{n},
\end{aligned}
$$

and

$$
\mathbf{e}=\left[e_{1}, e_{2}, \cdots, e_{n}\right]^{T} .
$$

From (4), (12), and (13), the tracking error dynamic equation can be expressed as

$$
\dot{\mathbf{e}}=\mathbf{A} \mathbf{e}+\mathbf{b}\left[y_{m}^{(n)}-f(\mathbf{x})-\Delta f(\mathbf{x})-m g(\mathbf{x}) v(t)-g(\mathbf{x}) d(v(t))\right],
$$

where

$$
\mathbf{A}=\left[\begin{array}{ccccccc}
0 & 1 & 0 & 0 & \cdots & 0 & 0 \\
0 & 0 & 1 & 0 & \cdots & 0 & 0 \\
\vdots & \vdots & \vdots & \vdots & \ddots & \vdots & \vdots \\
0 & 0 & 0 & 0 & \cdots & 0 & 1 \\
0 & 0 & 0 & 0 & \cdots & 0 & 0
\end{array}\right], \quad \mathbf{b}=\left[\begin{array}{c}
0 \\
0 \\
\vdots \\
0 \\
1
\end{array}\right] .
$$

Let $\mathbf{K}=\left[k_{1}, k_{2}, \cdots, k_{n}\right] \in R^{n}$ be chosen such that all roots of the polynomial $h(s)=s^{n}+k_{n} s^{n-1}+\cdots+k_{1}$ are in the open left-half plane. If the parameter $m$ and the functions $f$ and $g$ are known and the system is free of uncertainty, then control law of the certainty equivalent controller is obtained as

$$
v^{*}=\frac{1}{m g(\mathbf{x})}\left[\mathbf{K e}+y_{m}^{(n-1)}-f(\mathbf{x})-d(v(t))\right] .
$$

Substituting (15) into (14), we have

$$
e^{(n)}+k_{n} e^{(n-1)}+\cdots+k_{1} e=0,
$$

where the main objective of control is $\lim _{t \rightarrow \infty} e(t)=0$. However, in (15) some system parameter and functions such as $m, f$, and $g$ are unknown, and $\Delta f \neq 0$, the control input $v^{*}$ cannot be determined. In this situation, the approximation by fuzzy logic system can be employed to deal with this tracking control problem.

\subsection{Robust Adaptive Fuzzy Control Design}

Consider the tracking error dynamic equation (14) as follow:

$$
\dot{\mathbf{e}}=\mathbf{A e}+\mathbf{b}\left[y_{m}^{(n)}-f(\mathbf{x})-\Delta f(\mathbf{x})-m g(\mathbf{x}) v(t)-g(\mathbf{x}) d(v(t))\right] .
$$

Choose a matrix $\mathbf{K}=\left[k_{1}, k_{2}, \cdots, k_{n}\right] \in R^{n}$ such that $\mathbf{A}_{m}=\mathbf{A}-\mathbf{b K}$ is Hurwitz, and rewrite (16) as

$$
\dot{\mathbf{e}}=\mathbf{A}_{m} \mathbf{e}+\mathbf{b}\left[\mathbf{K e}+y_{m}^{(n)}-f(\mathbf{x})-\Delta f(\mathbf{x})-m g(\mathbf{x}) v(t)-g(\mathbf{x}) d(v(t))\right],
$$

where

$$
\mathbf{A}=\left[\begin{array}{ccccccc}
0 & 1 & 0 & 0 & \cdots & 0 & 0 \\
0 & 0 & 1 & 0 & \cdots & 0 & 0 \\
\vdots & \vdots & \vdots & \vdots & \ddots & \vdots & \vdots \\
0 & 0 & 0 & 0 & \cdots & 0 & 1 \\
0 & 0 & 0 & 0 & \cdots & 0 & 0
\end{array}\right], \mathbf{b}=\left[\begin{array}{l}
0 \\
0 \\
\vdots \\
0 \\
1
\end{array}\right], \mathbf{A}_{m}=\left[\begin{array}{ccccccc}
0 & 1 & 0 & 0 & \cdots & 0 & 0 \\
0 & 0 & 1 & 0 & \cdots & 0 & 0 \\
\vdots & \vdots & \vdots & \vdots & \ddots & \vdots & \vdots \\
0 & 0 & 0 & 0 & \cdots & 0 & 1 \\
-k_{1} & -k_{2} & -k_{3} & -k_{4} & \cdots & -k_{n-1} & -k_{n}
\end{array}\right]
$$

and $f$ and $g$ are unknown nonlinear functions and $\Delta f$ is the unknown uncertainty with the unknown upper bound function, i.e.,

$$
|\Delta f(\mathbf{x})| \leq h(\mathbf{x}) .
$$

where $h(\mathbf{x}(t))$ is an unknown smooth positive function and can be estimated by the adaptive law in the later.

First, let the nonlinear functions $f(\mathbf{x}), g(\mathbf{x})$, and $h(\mathbf{x})$ can be approximated, over a compact set $\Omega_{\mathbf{x}}$, by the fuzzy logic systems as follows:

$$
\begin{gathered}
\hat{f}\left(\mathbf{x} \mid \boldsymbol{\theta}_{f}\right)=\boldsymbol{\theta}_{f}^{T} \xi(\mathbf{x}), \\
\hat{g}\left(\mathbf{x} \mid \boldsymbol{\theta}_{g}\right)=\boldsymbol{\theta}_{g}^{T} \xi(\mathbf{x}), \\
\hat{h}\left(\mathbf{x} \mid \boldsymbol{\theta}_{h}\right)=\boldsymbol{\theta}_{h}^{T} \xi(\mathbf{x}),
\end{gathered}
$$

where $\xi(\mathbf{x})$ is the fuzzy basis vector, $\boldsymbol{\theta}_{f}, \boldsymbol{\theta}_{g}$, and $\boldsymbol{\theta}_{h}$ are the corresponding adjustable parameter vectors of each fuzzy logic system. Let us define the optimal parameter vectors $\boldsymbol{\theta}_{f}^{*}, \boldsymbol{\theta}_{g}^{*}$, and $\boldsymbol{\theta}_{h}^{*}$ as follows:

$$
\begin{gathered}
\boldsymbol{\theta}_{f}^{*}=\arg \min _{\boldsymbol{\theta}_{f} \in \Omega_{\boldsymbol{\theta}_{f}}}\left\{\sup _{\mathbf{x} \in \Omega_{\mathbf{x}}}\left|f(\mathbf{x})-\hat{f}\left(\mathbf{x} \mid \boldsymbol{\theta}_{f}\right)\right|\right\}, \\
\boldsymbol{\theta}_{g}^{*}=\arg \min _{\boldsymbol{\theta}_{g} \in \Omega_{\boldsymbol{\theta}_{g}}}\left\{\sup _{\mathbf{x} \in \Omega_{\mathbf{x}}}\left|g(\mathbf{x})-\hat{g}\left(\mathbf{x} \mid \boldsymbol{\theta}_{g}\right)\right|\right\}, \\
\boldsymbol{\theta}_{h}^{*}=\arg \min _{\boldsymbol{\theta}_{h} \in \Omega_{\boldsymbol{\theta}_{h}}}\left\{\sup _{\mathbf{x} \in \Omega_{\mathbf{x}}}\left|h(\mathbf{x})-\hat{h}\left(\mathbf{x} \mid \boldsymbol{\theta}_{h}\right)\right|\right\},
\end{gathered}
$$

where $\Omega_{\boldsymbol{\theta}_{f}}, \Omega_{\boldsymbol{\theta}_{g}}$, and $\Omega_{\boldsymbol{\theta}_{h}}$ denote the sets of suitable bounds on $\boldsymbol{\theta}_{f}, \boldsymbol{\theta}_{g}$, and $\boldsymbol{\theta}_{h}$, respectively. Also the parameter estimation errors are defined as

$$
\begin{aligned}
& \tilde{\boldsymbol{\theta}}_{f}=\boldsymbol{\theta}_{f}-\boldsymbol{\theta}_{f}^{*}, \quad \tilde{\boldsymbol{\theta}}_{g}=\boldsymbol{\theta}_{g}-\boldsymbol{\theta}_{g}^{*}, \quad \tilde{\boldsymbol{\theta}}_{h}=\boldsymbol{\theta}_{h}-\boldsymbol{\theta}_{h}^{*}, \\
& \text { and } \\
& \omega_{1}=\left(f(\mathbf{x})-\hat{f}\left(\mathbf{x} \mid \boldsymbol{\theta}_{f}^{*}\right)\right)+\left(g(\mathbf{x})-\hat{g}\left(\mathbf{x} \mid \boldsymbol{\theta}_{g}^{*}\right)\right) w(t), \\
& \omega_{2}=h(\mathbf{x})-h\left(\mathbf{x} \mid \boldsymbol{\theta}_{h}^{*}\right),
\end{aligned}
$$

as the minimum approximation errors, which correspond to approximation errors obtained when optimal parameters are used.

Secondly, we define

$$
\tilde{\phi}=\hat{\phi}-\phi
$$

where $\hat{\phi}$ is an estimate of $\phi$, which is defined as 
$\phi:=(m)^{-1}$.

Based on the given plant and dead-zone models under the assumptions 1-3, consider the following controller:

$$
v=v_{1}+v_{2}+v_{3} \text {, }
$$

where

$$
\begin{aligned}
& v_{1}=\frac{1}{\hat{g}\left(\mathbf{x} \mid \boldsymbol{\theta}_{g}\right)} \cdot \hat{\phi} \cdot\left(\mathbf{K e}+y_{m}^{(n)}-\hat{f}\left(\mathbf{x} \mid \boldsymbol{\theta}_{f}\right)\right), \\
& v_{2}=\frac{1}{\hat{g}\left(\mathbf{x} \mid \boldsymbol{\theta}_{g}\right)} \cdot \hat{\phi} \cdot \hat{h}\left(\mathbf{x} \mid \boldsymbol{\theta}_{h}\right) \cdot \operatorname{sgn}\left(\mathbf{e}^{T} \mathbf{P b}\right), \\
& v_{3}=k^{*} \cdot \operatorname{sgn}\left(\hat{g}\left(\mathbf{x} \mid \boldsymbol{\theta}_{g}\right)\right) \cdot \operatorname{sgn}\left(\mathbf{e}^{T} \mathbf{P b}\right),
\end{aligned}
$$

where $k^{*} \geq \rho / m_{\min }, \rho$ is defined in (6), and $\mathbf{P}$ is a positive definite matrix, which is a solution of the following Lyapunov equation

$$
\mathbf{A}_{m}^{T} \mathbf{P}+\mathbf{P} \mathbf{A}_{m}=-\mathbf{Q}
$$

where $\mathbf{Q}$ is a positive definite matrix, and the parameter update laws are as follows:

$$
\begin{array}{r}
\dot{\hat{\phi}}=\eta \cdot\left[\left\|\mathbf{e}^{T} \mathbf{P b}\right\| \cdot \hat{h}\left(\mathbf{x} \mid \boldsymbol{\theta}_{h}\right)+\mathbf{e}^{T} \mathbf{P b} \cdot\left(\mathbf{K e}+y_{m}^{(n)}-\hat{f}\left(\mathbf{x} \mid \boldsymbol{\theta}_{f}\right)\right)\right] \\
\dot{\boldsymbol{\theta}}_{f}=-\gamma_{f} \cdot \mathbf{e}^{T} \mathbf{P b} \cdot \xi(\mathbf{x}) \\
\dot{\boldsymbol{\theta}}_{g}=-\gamma_{g} \cdot \mathbf{e}^{T} \mathbf{P b} \cdot \xi(\mathbf{x}) \cdot\left(v(t)+d_{1}(v(t))\right) \\
\dot{\boldsymbol{\theta}}_{h}=\gamma_{h} \cdot\left\|\mathbf{e}^{T} \mathbf{P b}\right\| \cdot \xi(\mathbf{x})
\end{array}
$$

where $\eta, \gamma_{f}, \gamma_{g}$, and $\gamma_{h}$ are positive constants, determining the rates of adaptations, and

$$
d_{1}(v(t))= \begin{cases}-b_{r} & \text { for } v(t) \geq b_{r}, \\ -v(t) & \text { for } b_{l}<v(t)<b_{r}, \\ -b_{l} & \text { for } v(t) \leq b_{l} .\end{cases}
$$

The main result of the robust adaptive fuzzy control scheme is summarized in the following theorem.

Theorem 1. Consider the uncertain nonlinear system (1) with an unknown dead-zone (3) at the input subject to the assumptions 1-3. The robust adaptive fuzzy controller defined by (28)-(31) with adaptation laws given by (33)-(36) ensures that all the closed-loop signals are bounded, and the tracking errors converge to a neighborhood of zero.

Proof. Consider the Lyapunov function candidate

$$
V=\frac{1}{2}\left(\frac{1}{m} \mathbf{e}^{T} \mathbf{P e}+\frac{1}{m \cdot \gamma_{f}} \tilde{\boldsymbol{\theta}}_{f}^{T} \tilde{\boldsymbol{\theta}}_{f}+\frac{1}{\gamma_{g}} \tilde{\boldsymbol{\theta}}_{g}^{T} \tilde{\boldsymbol{\theta}}_{g}+\frac{1}{m \cdot \gamma_{h}} \tilde{\boldsymbol{\theta}}_{h}^{T} \tilde{\boldsymbol{\theta}}_{h}+\frac{1}{\eta} \tilde{\phi}^{2}\right) .
$$

Differentiating the Lyapunov function $V$ with respect to time, we can obtain

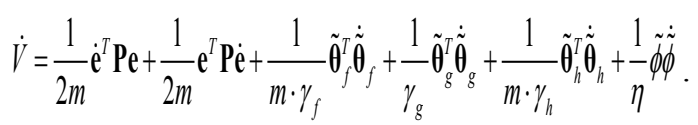

From (17) and by the fact $\dot{\tilde{\boldsymbol{\theta}}}_{f}=\dot{\boldsymbol{\theta}}_{f}, \quad \dot{\tilde{\boldsymbol{\theta}}}_{g}=\dot{\boldsymbol{\theta}}_{g}$, $\dot{\tilde{\boldsymbol{\theta}}}_{h}=\dot{\boldsymbol{\theta}}_{h}$, and $\dot{\tilde{\phi}}=\dot{\hat{\phi}}$, the above equation becomes

$$
\begin{aligned}
\dot{V} & =\frac{1}{2 m}\left\{\mathbf{A}_{m} \mathbf{e}+\mathbf{b}\left[\mathbf{K e}+y_{m}^{(n)}-f(\mathbf{x})-\Delta f(\mathbf{x})-m g(\mathbf{x}) v(t)-g(\mathbf{x}) d(v(t))\right]\right\}^{T} \mathbf{P e} \\
& +\frac{1}{2 m} \mathbf{e}^{T} \mathbf{P}\left\{\mathbf{A}_{m} \mathbf{e}+\mathbf{b}\left[\begin{array}{l}
\mathbf{K e}+y_{m}^{(n)}-f(\mathbf{x})-\Delta f(\mathbf{x}) \\
-m g(\mathbf{x}) v(t)-g(\mathbf{x}) d(v(t))
\end{array}\right]\right\}+\frac{1}{m \cdot \gamma_{f}} \tilde{\boldsymbol{\theta}}_{f}^{T} \dot{\boldsymbol{\theta}}_{f}+\frac{1}{\gamma_{g}} \tilde{\boldsymbol{\theta}}_{g}^{T} \dot{\boldsymbol{\theta}}_{g}+\frac{1}{m \cdot \gamma_{h}} \tilde{\boldsymbol{\theta}}_{h}^{T} \dot{\boldsymbol{\theta}}_{h}+\frac{1}{\eta} \tilde{\phi} \dot{\hat{\phi}} \\
& =\frac{1}{2 m} \mathbf{e}^{T}\left[\mathbf{A}_{m}^{T} \mathbf{P}+\mathbf{P} \mathbf{A}_{m}\right] \mathbf{e}+\frac{1}{m} \mathbf{e}^{T} \mathbf{P b}\left[\mathbf{K e}+y_{m}^{(n)}-f(\mathbf{x})-\Delta f(\mathbf{x})-m g(\mathbf{x}) v(t)-g(\mathbf{x}) d(v(t))\right] \\
& +\frac{1}{m \cdot \gamma_{f}} \tilde{\boldsymbol{\theta}}_{f}^{T} \dot{\boldsymbol{\theta}}_{f}+\frac{1}{\gamma_{g}} \tilde{\boldsymbol{\theta}}_{g}^{T} \dot{\boldsymbol{\theta}}_{g}+\frac{1}{m \cdot \gamma_{h}} \tilde{\boldsymbol{\theta}}_{h}^{T} \dot{\boldsymbol{\theta}}_{h}+\frac{1}{\eta} \tilde{\phi} \dot{\hat{\phi}} .
\end{aligned}
$$

Applying (32) and (18) to (40) yields

$$
\begin{aligned}
& \dot{V}=-\frac{1}{2 m} \mathbf{e}^{T} \mathbf{Q e}+\frac{1}{m} \mathbf{e}^{T} \mathbf{P b}\left[\mathbf{K e}+y_{m}^{(n)}-f(\mathbf{x})-m g(\mathbf{x}) v(t)-g(\mathbf{x}) d(v(t))\right]-\frac{1}{m} \mathbf{e}^{T} \mathbf{P b} \cdot \Delta f(\mathbf{x})+\frac{1}{m \cdot \gamma_{f}} \tilde{\boldsymbol{\theta}}_{f}^{T} \dot{\boldsymbol{\theta}}_{f}+\frac{1}{\gamma_{g}} \tilde{\boldsymbol{\theta}}_{g}^{T} \dot{\boldsymbol{\theta}}_{g}+\frac{1}{m \cdot \gamma_{h}} \tilde{\boldsymbol{\theta}}_{h}^{T} \dot{\boldsymbol{\theta}}_{h}+\frac{1}{\eta} \tilde{\phi} \dot{\hat{\phi}} \\
\leq & -\frac{1}{2 m} \mathbf{e}^{T} \mathbf{Q e}+\frac{1}{m} \mathbf{e}^{T} \mathbf{P b}\left[\mathbf{K e}+y_{m}^{(n)}-f(\mathbf{x})-m g(\mathbf{x}) v(t)-g(\mathbf{x}) d(v(t))\right]+\frac{1}{|m|}\left\|\mathbf{e}^{T} \mathbf{P b}\right\| \cdot|\Delta f(\mathbf{x})|+\frac{1}{m \cdot \gamma_{f}} \tilde{\boldsymbol{\theta}}_{f}^{T} \dot{\boldsymbol{\theta}}_{f}+\frac{1}{\gamma_{g}} \tilde{\boldsymbol{\theta}}_{g}^{T} \dot{\boldsymbol{\theta}}_{g}+\frac{1}{m \cdot \gamma_{h}} \tilde{\boldsymbol{\theta}}_{h}^{T} \dot{\boldsymbol{\theta}}_{h}+\frac{1}{\eta} \tilde{\phi} \dot{\hat{\phi}} \\
\leq & -\frac{1}{2 m} \mathbf{e}^{T} \mathbf{Q e}+\frac{1}{m} \mathbf{e}^{T} \mathbf{P b}\left[\mathbf{K e}+y_{m}^{(n)}-f(\mathbf{x})-m g(\mathbf{x}) v(t)-g(\mathbf{x}) d(v(t))\right]+\frac{1}{m}\left\|\mathbf{e}^{T} \mathbf{P b}\right\| \cdot h(\mathbf{x})+\frac{1}{m \cdot \gamma_{f}} \tilde{\boldsymbol{\theta}}_{f}^{T} \dot{\boldsymbol{\theta}}_{f}+\frac{1}{\gamma_{g}} \tilde{\boldsymbol{\theta}}_{g}^{T} \dot{\boldsymbol{\theta}}_{g}+\frac{1}{m \cdot \gamma_{h}} \tilde{\boldsymbol{\theta}}_{h}^{T} \dot{\boldsymbol{\theta}}_{h}+\frac{1}{\eta} \tilde{\phi} \dot{\hat{\phi}} .
\end{aligned}
$$

According to (19)-(26), we obtain 


$$
\begin{aligned}
& \dot{V} \leq-\frac{1}{2 m} \mathbf{e}^{T} \mathbf{Q} \mathbf{e}+\frac{1}{m} \mathbf{e}^{T} \mathbf{P b}\left[\mathbf{K e}+y_{m}^{(n)}-\hat{f}\left(\mathbf{x} \mid \boldsymbol{\theta}_{f}\right)-\left(f(\mathbf{x})-\hat{f}\left(\mathbf{x} \mid \boldsymbol{\theta}_{f}\right)\right)-\hat{g}\left(\mathbf{x} \mid \boldsymbol{\theta}_{g}\right)(m v(t)+d(v(t)))-\left(g(\mathbf{x})-\hat{g}\left(\mathbf{x} \mid \boldsymbol{\theta}_{g}\right)\right)(m v(t)+d(v(t)))\right] \\
& +\frac{1}{m}\left\|\mathbf{e}^{T} \mathbf{P b}\right\| \cdot \hat{h}\left(\mathbf{x} \mid \boldsymbol{\theta}_{h}\right)+\frac{1}{m}\left\|\mathbf{e}^{T} \mathbf{P b}\right\| \cdot\left(h(\mathbf{x})-\hat{h}\left(\mathbf{x} \mid \boldsymbol{\theta}_{h}\right)\right)+\frac{1}{m \cdot \gamma_{f}} \tilde{\boldsymbol{\theta}}_{f}^{T} \dot{\boldsymbol{\theta}}_{f}+\frac{1}{\gamma_{g}} \tilde{\boldsymbol{\theta}}_{g}^{T} \dot{\boldsymbol{\theta}}_{g}+\frac{1}{m \cdot \gamma_{h}} \tilde{\boldsymbol{\theta}}_{h}^{T} \dot{\boldsymbol{\theta}}_{h}+\frac{1}{\eta} \tilde{\phi} \dot{\hat{\phi}} \\
& =-\frac{1}{2 m} \mathbf{e}^{T} \mathbf{Q e}+\frac{1}{m} \mathbf{e}^{T} \mathbf{P b}\left[\mathbf{K e}+y_{m}^{(n)}-\hat{f}\left(\mathbf{x} \mid \boldsymbol{\theta}_{f}\right)-\left(f\left(\mathbf{x} \mid \boldsymbol{\theta}_{f}^{*}\right)-\hat{f}\left(\mathbf{x} \mid \boldsymbol{\theta}_{f}\right)\right)-\omega_{1}-\hat{g}\left(\mathbf{x} \mid \boldsymbol{\theta}_{g}\right)(m v(t)+d(v(t)))-\left(g\left(\mathbf{x} \mid \boldsymbol{\theta}_{g}^{*}\right)-\hat{g}\left(\mathbf{x} \mid \boldsymbol{\theta}_{g}\right)\right)(m v(t)+d(v(t)))\right] \\
& +\frac{1}{m}\left\|\mathbf{e}^{T} \mathbf{P b}\right\| \cdot \hat{h}\left(\mathbf{x} \mid \boldsymbol{\theta}_{h}\right)+\frac{1}{m}\left\|\mathbf{e}^{T} \mathbf{P b}\right\| \cdot\left(h\left(\mathbf{x} \mid \boldsymbol{\theta}_{h}^{*}\right)-\hat{h}\left(\mathbf{x} \mid \boldsymbol{\theta}_{h}\right)\right)+\frac{1}{m}\left\|\mathbf{e}^{T} \mathbf{P b}\right\| \cdot \omega_{2}+\frac{1}{m \cdot \gamma_{f}} \tilde{\boldsymbol{\theta}}_{f}^{T} \dot{\boldsymbol{\theta}}_{f}+\frac{1}{\gamma_{g}} \tilde{\boldsymbol{\theta}}_{g}^{T} \dot{\boldsymbol{\theta}}_{g}+\frac{1}{m \cdot \gamma_{h}} \tilde{\boldsymbol{\theta}}_{h}^{T} \dot{\boldsymbol{\theta}}_{h}+\frac{1}{\eta} \tilde{\phi} \dot{\hat{\phi}} \\
& =-\frac{1}{2 m} \mathbf{e}^{T} \mathbf{Q} \mathbf{e}-\frac{1}{m} \mathbf{e}^{T} \mathbf{P b}\left(f\left(\mathbf{x} \mid \boldsymbol{\theta}_{f}^{*}\right)-\hat{f}\left(\mathbf{x} \mid \boldsymbol{\theta}_{f}\right)\right)+\frac{1}{m} \mathbf{e}^{T} \mathbf{P b}\left[\mathbf{K e}+y_{m}^{(n)}-\hat{f}\left(\mathbf{x} \mid \boldsymbol{\theta}_{f}\right)-\hat{g}\left(\mathbf{x} \mid \boldsymbol{\theta}_{g}\right)(m v(t)+d(v(t)))\right] \\
& -\frac{1}{m} \mathbf{e}^{T} \mathbf{P b}\left(g\left(\mathbf{x} \mid \boldsymbol{\theta}_{g}^{*}\right)-\hat{g}\left(\mathbf{x} \mid \boldsymbol{\theta}_{g}\right)\right)(m v(t)+d(v(t)))-\frac{1}{m} \mathbf{e}^{T} \mathbf{P b} \cdot \omega_{1}+\frac{1}{m}\left\|\mathbf{e}^{T} \mathbf{P b}\right\| \cdot \hat{h}\left(\mathbf{x} \mid \boldsymbol{\theta}_{h}\right)+\frac{1}{m}\left\|\mathbf{e}^{T} \mathbf{P b}\right\| \cdot\left(h\left(\mathbf{x} \mid \boldsymbol{\theta}_{h}^{*}\right)-\hat{h}\left(\mathbf{x} \mid \boldsymbol{\theta}_{h}\right)\right)+\frac{1}{m}\left\|\mathbf{e}^{T} \mathbf{P b}\right\| \cdot \omega_{2} \\
& +\frac{1}{m \cdot \gamma_{f}} \tilde{\boldsymbol{\theta}}_{f}^{T} \dot{\boldsymbol{\theta}}_{f}+\frac{1}{\gamma_{g}} \tilde{\boldsymbol{\theta}}_{g}^{T} \dot{\boldsymbol{\theta}}_{g}+\frac{1}{m \cdot \gamma_{h}} \tilde{\boldsymbol{\theta}}_{h}^{T} \dot{\boldsymbol{\theta}}_{h}+\frac{1}{\eta} \tilde{\phi} \dot{\hat{\phi}} \\
& =-\frac{1}{2 m} \mathbf{e}^{T} \mathbf{Q} \mathbf{e}-\frac{1}{m} \mathbf{e}^{T} \mathbf{P b}\left(\boldsymbol{\theta}_{f}^{* T} \xi(\mathbf{x})-\boldsymbol{\theta}_{f}^{T} \xi(\mathbf{x})\right)+\frac{1}{m} \mathbf{e}^{T} \mathbf{P b}\left[\mathbf{K e}+y_{m}^{(n)}-\hat{f}\left(\mathbf{x} \mid \boldsymbol{\theta}_{f}\right)-m \hat{g}\left(\mathbf{x} \mid \boldsymbol{\theta}_{g}\right) v(t)-\hat{g}\left(\mathbf{x} \mid \boldsymbol{\theta}_{g}\right) d(v(t))\right] \\
& -\frac{1}{m} \mathbf{e}^{T} \mathbf{P b}\left(\boldsymbol{\theta}_{g}^{* T} \xi(\mathbf{x})-\boldsymbol{\theta}_{g}^{T} \xi(\mathbf{x})\right)\left(m v(t)+m \cdot d_{1}(v(t))\right)-\frac{1}{m} \mathbf{e}^{T} \mathbf{P b} \cdot \omega_{1}+\frac{1}{m}\left\|\mathbf{e}^{T} \mathbf{P b}\right\| \cdot \hat{h}\left(\mathbf{x} \mid \boldsymbol{\theta}_{h}\right)+\frac{1}{m}\left\|\mathbf{e}^{T} \mathbf{P b}\right\| \cdot\left(\boldsymbol{\theta}_{h}^{* T} \xi(\mathbf{x})-\boldsymbol{\theta}_{h}^{T} \xi(\mathbf{x})\right)+\frac{1}{m}\left\|\mathbf{e}^{T} \mathbf{P b}\right\| \cdot \omega_{2} \\
& +\frac{1}{m \cdot \gamma_{f}} \tilde{\boldsymbol{\theta}}_{f}^{T} \dot{\boldsymbol{\theta}}_{f}+\frac{1}{\gamma_{g}} \tilde{\boldsymbol{\theta}}_{g}^{T} \dot{\boldsymbol{\theta}}_{g}+\frac{1}{m \cdot \gamma_{h}} \tilde{\boldsymbol{\theta}}_{h}^{T} \dot{\boldsymbol{\theta}}_{h}+\frac{1}{\eta} \tilde{\phi} \dot{\hat{\phi}} \\
& =-\frac{1}{2 m} \mathbf{e}^{T} \mathbf{Q e}+\frac{1}{m} \mathbf{e}^{T} \mathbf{P b} \cdot \tilde{\boldsymbol{\theta}}_{f}^{T} \xi(\mathbf{x})+\frac{1}{m} \mathbf{e}^{T} \mathbf{P b}\left[\mathbf{K e}+y_{m}^{(n)}-\hat{f}\left(\mathbf{x} \mid \boldsymbol{\theta}_{f}\right)-m \hat{g}\left(\mathbf{x} \mid \boldsymbol{\theta}_{g}\right) v(t)-\hat{g}\left(\mathbf{x} \mid \boldsymbol{\theta}_{g}\right) d(v(t))\right] \\
& +\frac{1}{m} \mathbf{e}^{T} \mathbf{P b} \cdot \tilde{\boldsymbol{\theta}}_{g}^{T} \xi(\mathbf{x})\left(m v(t)+m \cdot d_{1}(v(t))\right)-\frac{1}{m} \mathbf{e}^{T} \mathbf{P b} \cdot \omega_{1}+\frac{1}{m}\left\|\mathbf{e}^{T} \mathbf{P b}\right\| \cdot \hat{h}\left(\mathbf{x} \mid \boldsymbol{\theta}_{h}\right)-\frac{1}{m}\left\|\mathbf{e}^{T} \mathbf{P b}\right\| \cdot \tilde{\boldsymbol{\theta}}_{h}^{T} \xi(\mathbf{x})+\frac{1}{m}\left\|\mathbf{e}^{T} \mathbf{P b}\right\| \cdot \omega_{2} \\
& +\frac{1}{m \cdot \gamma_{f}} \tilde{\boldsymbol{\theta}}_{f}^{T} \dot{\boldsymbol{\theta}}_{f}+\frac{1}{\gamma_{g}} \tilde{\boldsymbol{\theta}}_{g}^{T} \dot{\boldsymbol{\theta}}_{g}+\frac{1}{m \cdot \gamma_{h}} \tilde{\boldsymbol{\theta}}_{h}^{T} \dot{\boldsymbol{\theta}}_{h}+\frac{1}{\eta} \tilde{\phi} \dot{\hat{\phi}} .
\end{aligned}
$$

Substituting (34)-(36) into (42), we have

$$
\begin{aligned}
\dot{V} \leq & -\frac{1}{2 m} \mathbf{e}^{T} \mathbf{Q e}-\frac{1}{m} \mathbf{e}^{T} \mathbf{P b} \cdot \omega_{1}+\frac{1}{m}\left\|\mathbf{e}^{T} \mathbf{P b}\right\| \cdot \omega_{2} \\
& +\frac{1}{m} \mathbf{e}^{T} \mathbf{P b}\left[\mathbf{K e}+y_{m}^{(n)}-\hat{f}\left(\mathbf{x} \mid \boldsymbol{\theta}_{f}\right)-m \hat{g}\left(\mathbf{x} \mid \boldsymbol{\theta}_{g}\right) v(t)-\hat{g}\left(\mathbf{x} \mid \boldsymbol{\theta}_{g}\right) d(v(t))\right]+\frac{1}{m}\left\|\mathbf{e}^{T} \mathbf{P b}\right\| \cdot \hat{h}\left(\mathbf{x} \mid \boldsymbol{\theta}_{h}\right)+\frac{1}{\eta} \tilde{\phi} \dot{\hat{\phi}} \\
= & -\frac{1}{2 m} \mathbf{e}^{T} \mathbf{Q e}-\frac{1}{m} \mathbf{e}^{T} \mathbf{P b} \cdot \omega_{1}+\frac{1}{m}\left\|\mathbf{e}^{T} \mathbf{P b}\right\| \cdot \omega_{2} \\
& +\mathbf{e}^{T} \mathbf{P b}\left[\frac{1}{m}\left(\mathbf{K e}+y_{m}^{(n)}-\hat{f}\left(\mathbf{x} \mid \boldsymbol{\theta}_{f}\right)\right)-\hat{g}\left(\mathbf{x} \mid \boldsymbol{\theta}_{g}\right) v(t)-\hat{g}\left(\mathbf{x} \mid \boldsymbol{\theta}_{g}\right) \frac{d(v(t))}{m}\right]+\frac{1}{m}\left\|\mathbf{e}^{T} \mathbf{P b}\right\| \cdot \hat{h}\left(\mathbf{x} \mid \boldsymbol{\theta}_{h}\right)+\frac{1}{\eta} \tilde{\phi} \dot{\phi} \\
= & -\frac{1}{2 m} \mathbf{e}^{T} \mathbf{Q e}-\frac{1}{m} \mathbf{e}^{T} \mathbf{P b} \cdot \omega_{1}+\frac{1}{m}\left\|\mathbf{e}^{T} \mathbf{P b}\right\| \cdot \omega_{2} \\
& +\mathbf{e}^{T} \mathbf{P b}\left[\phi \cdot\left(\mathbf{K e}+y_{m}^{(n)}-\hat{f}\left(\mathbf{x} \mid \boldsymbol{\theta}_{f}\right)\right)-\hat{g}\left(\mathbf{x} \mid \boldsymbol{\theta}_{g}\right) v(t)\right]-\mathbf{e}^{T} \mathbf{P b} \cdot \hat{g}\left(\mathbf{x} \mid \boldsymbol{\theta}_{g}\right) \frac{d(v(t))}{m}+\phi \cdot\left\|\mathbf{e}^{T} \mathbf{P b}\right\| \cdot \hat{h}\left(\mathbf{x} \mid \boldsymbol{\theta}_{h}\right)+\frac{1}{\eta} \tilde{\phi} \dot{\hat{\phi}} \\
\leq & -\frac{1}{2 m} \mathbf{e}^{T} \mathbf{Q} \mathbf{e}-\frac{1}{m} \mathbf{e}^{T} \mathbf{P b} \cdot \omega_{1}+\frac{1}{m}\left\|\mathbf{e}^{T} \mathbf{P b}\right\| \cdot \omega_{2} \\
& +\mathbf{e}^{T} \mathbf{P b}\left[\phi \cdot\left(\mathbf{K e}+y_{m}^{(n)}-\hat{f}\left(\mathbf{x} \mid \boldsymbol{\theta}_{f}\right)\right)-\hat{g}\left(\mathbf{x} \mid \boldsymbol{\theta}_{g}\right) v(t)\right]+\left\|\mathbf{e}^{T} \mathbf{P b}\right\| \cdot\left|\hat{g}\left(\mathbf{x} \mid \boldsymbol{\theta}_{g}\right)\right| \frac{d(v(t)) \mid}{|m|}+\phi \cdot\left\|\mathbf{e}^{T} \mathbf{P b}\right\| \cdot \hat{h}\left(\mathbf{x} \mid \boldsymbol{\theta}_{h}\right)+\frac{1}{\eta} \tilde{\phi} \dot{\hat{\phi}} .
\end{aligned}
$$

Using the control law (28)-(31), the above equation can be rewritten as 


$$
\begin{aligned}
\dot{V} \leq & -\frac{1}{2 m} \mathbf{e}^{T} \mathbf{Q e}-\frac{1}{m} \mathbf{e}^{T} \mathbf{P b} \cdot \omega_{1}+\frac{1}{m}\left\|\mathbf{e}^{T} \mathbf{P b}\right\| \cdot \omega_{2}+\mathbf{e}^{T} \mathbf{P b}\left[\phi \cdot\left(\mathbf{K e}+y_{m}^{(n)}-\hat{f}\left(\mathbf{x} \mid \boldsymbol{\theta}_{f}\right)\right)-\hat{\phi} \cdot\left(\mathbf{K e}+y_{m}^{(n)}-\hat{f}\left(\mathbf{x} \mid \boldsymbol{\theta}_{f}\right)\right)\right] \\
& -\left\|\mathbf{e}^{T} \mathbf{P b}\right\| \cdot \mid \hat{g}\left(\mathbf{x} \mid \boldsymbol{\theta}_{g}\right)\left\|\left(k^{*}-\frac{\mid d(v(t))) \mid}{|m|}\right)+(\phi-\hat{\phi}) \cdot\right\| \mathbf{e}^{T} \mathbf{P b} \| \cdot \hat{h}\left(\mathbf{x} \mid \boldsymbol{\theta}_{g}\right)+\frac{1}{\eta} \tilde{\phi} \dot{\hat{\phi}} \\
& \leq-\frac{1}{2 m} \mathbf{e}^{T} \mathbf{Q e}-\frac{1}{m} \mathbf{e}^{T} \mathbf{P b} \cdot \omega_{1}+\frac{1}{m}\left\|\mathbf{e}^{T} \mathbf{P b}\right\| \cdot \omega_{2}-\tilde{\phi} \cdot \mathbf{e}^{T} \mathbf{P b} \cdot\left(\mathbf{K e}+y_{m}^{(n)}-\hat{f}\left(\mathbf{x} \mid \boldsymbol{\theta}_{f}\right)\right)-\tilde{\phi} \cdot\left\|\mathbf{e}^{T} \mathbf{P b}\right\| \cdot \hat{h}\left(\mathbf{x} \mid \boldsymbol{\theta}_{g}\right)+\frac{1}{\eta} \tilde{\phi} \dot{\hat{\phi}} .
\end{aligned}
$$

By applying adaptation law (33), one has

$$
\begin{aligned}
\dot{V} & \leq-\frac{1}{2 m} \mathrm{e}^{T} \mathbf{Q} \mathrm{e}-\frac{1}{m} \mathrm{e}^{T} \mathbf{P b} \cdot \omega_{1}+\frac{1}{m}\left\|\mathrm{e}^{T} \mathbf{P b}\right\| \cdot \omega_{2} \\
& \approx-\frac{1}{2 m} \mathrm{e}^{T} \mathbf{Q} .
\end{aligned}
$$

Therefore, it can be concluded that $\dot{V} \leq 0$ from (45), and the closed-loop system is asymptotically stable based on Lyapunov synthesis approach. This completes the proof.

\section{An Example and Simulation Results}

Consider the second-order nonlinear system

$$
\begin{aligned}
& \dot{x}_{1}=x_{2}, \\
& \dot{x}_{2}=\frac{1-e^{-x_{1}}}{1+e^{-x_{1}}}-0.5 x_{1} \sin (3 t)+w(t), \\
& y=x_{1},
\end{aligned}
$$

where the nonlinear functions $f(\mathbf{x})=\left(1-e^{-x_{1}}\right) /\left(1+e^{-x_{1}}\right)$ and $g(\mathbf{x})=1$ are assumed to be unknown, and the $\Delta f(\mathbf{x})=-0.5 x_{1} \sin (3 t)$ is an unknown uncertain with unknown upper bound functions, i.e., $|\Delta f(\mathbf{x})| \leq h(\mathbf{x})$, and $w(t)$ is an output of a dead-zone. The control objective is to maintain the system output $y$ to follow the reference signal $y_{m}=2.5 \sin (t)$.

In the simulation, parameters of the dead-zone are $m=1$, $b_{r}=0.5, b_{l}=-0.6$. And their bounds are chosen as $m_{\text {max }}=1.25, m_{\text {min }}=0.85, b_{r \text { max }}=0.6, b_{r \text { min }}=0.1$, $b_{l \text { max }}=-0.1, b_{l \text { min }}=-0.7$, and $k^{*}=2.5$. In the implementation, six fuzzy sets are defined over interval[-3, 3] for both $x_{1}$ and $x_{2}$, with labels $N B, N M, N S, P S, P M$, and $P B$, and their membership functions are

$$
\begin{aligned}
& \mu_{N B}\left(x_{i}\right)=\frac{1}{1+\exp \left(5\left(x_{i}+2\right)\right)}, \\
& \mu_{N M}\left(x_{i}\right)=\exp \left(-\left(x_{i}+1.5\right)^{2}\right), \\
& \mu_{N S}\left(x_{i}\right)=\exp \left(-\left(x_{i}+0.5\right)^{2}\right),
\end{aligned}
$$

$$
\begin{gathered}
\mu_{P S}\left(x_{i}\right)=\exp \left(-\left(x_{i}-0.5\right)^{2}\right), \\
\mu_{P M}\left(x_{i}\right)=\exp \left(-\left(x_{i}-1.5\right)^{2}\right), \\
\mu_{P B}\left(x_{i}\right)=\frac{1}{1+\exp \left(5\left(x_{i}-2\right)\right)}, \quad i=1,2 .
\end{gathered}
$$

In this section, we apply the proposed robust adaptive fuzzy control approach in Section 3 to deal with the above second-order nonlinear system. Let $\mathbf{K}=[1,2]$ (so that $p^{2}+k_{2} p+k_{1}$ is stable) and $\mathbf{Q}=\operatorname{diag}[10,10]$, then we have Lyapunov equation (32) and obtain

$$
\mathbf{P}=\left[\begin{array}{cc}
15 & 5 \\
5 & 5
\end{array}\right] .
$$

Choose the initial values are chosen as $\mathbf{x}(0)=[-2.5,3.5]$, $\boldsymbol{\theta}_{f}(0)=\mathbf{0}, \boldsymbol{\theta}_{g}(0)=\mathbf{5}, \theta_{h}(0)=\mathbf{0}$, and $\hat{\phi}(0)=0.85$, and $\gamma_{f}=1.5, \gamma_{g}=2.0, \gamma_{h}=1.5$, and $\eta=1.0$, and boundary layer $\varepsilon=0.13$. Simulation results are shown in Figs. 3-5. Fig. 3 shows the position tracking performance. Fig 4 shows the velocity tracking performance. The control signal is shown in Fig. 5. Apparently, the proposed control scheme can achieve the objective of good tracking performance and robust stability simultaneously.

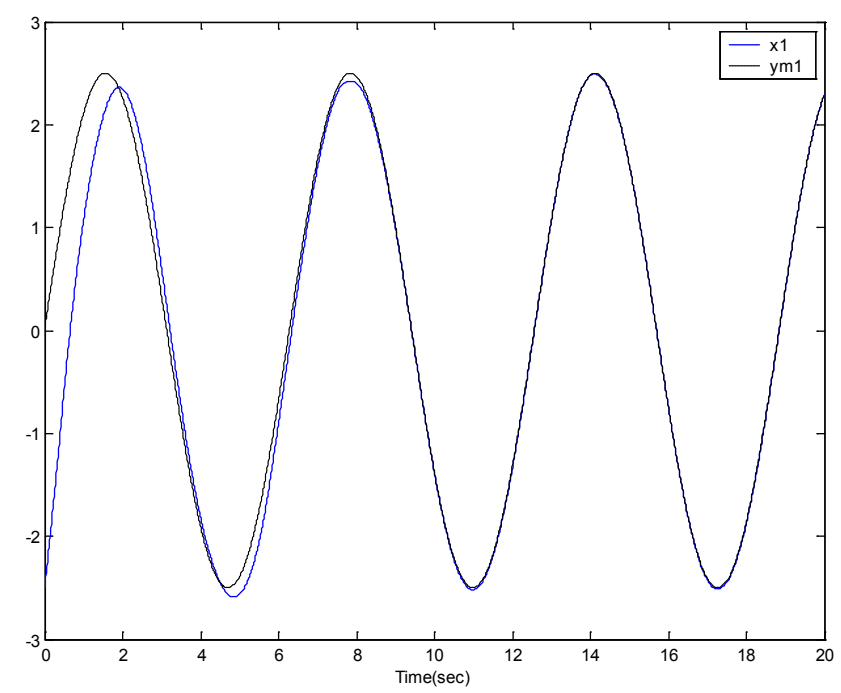

Figure 3. The output $y\left(=x_{1}\right)$ (solid line) and its desired value $y_{m}$ (dashed line) 


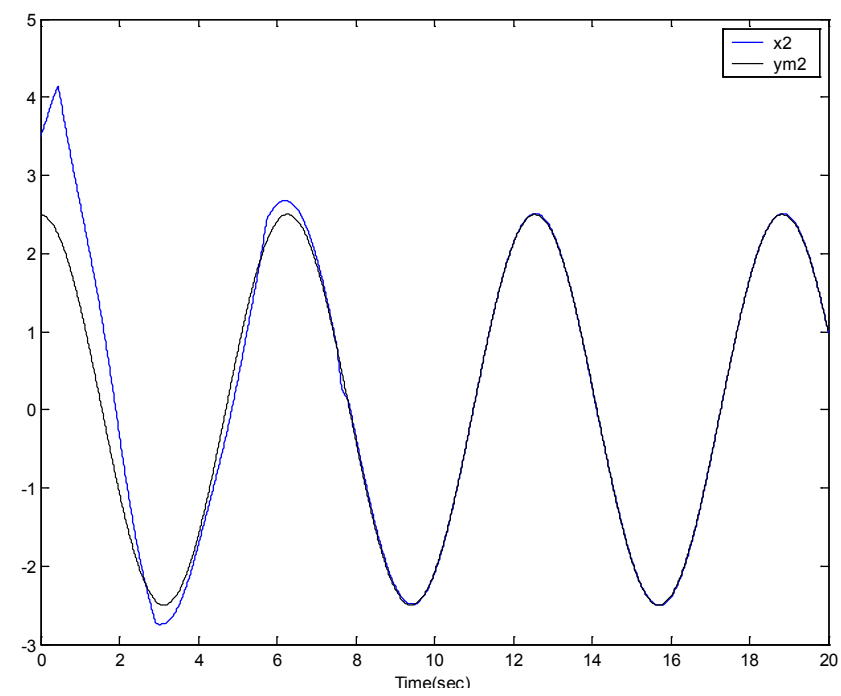

Figure 4. The output $\dot{y}\left(=x_{2}\right)$ (solid line) and its desired value $\dot{y}_{m}$ (dashed line)

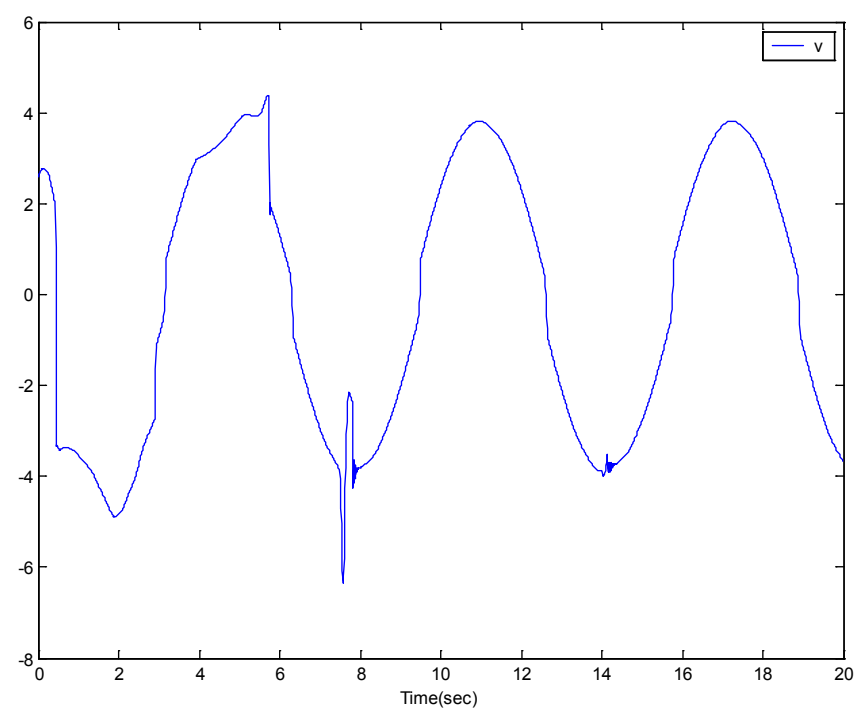

Figure 5. The control input $v$

\section{Conclusions}

Dead-zone with unknown parameters in physical components may severely limit the performance of control, and its characteristics are quite commonly encountered in actuators in practical control systems. By using a description of a dead-zone and exploring the exploring the properties of this dead-zone model intuitively and mathematically, this paper presents the robust adaptive fuzzy control scheme without constructing the dead-zone inverse for a class of uncertain nonlinear systems containing an unknown dead-zone. Based on Lyapunov stability theorem, the proposed robust adaptive fuzzy control scheme can not only guarantee the robust stability of the whole closed-loop system with an unknown dead-zone in the actuator, but also obtain the good tracking performance. Finally, simulation results are illustrated to verify the effectiveness of the proposed method.

\section{REFERENCES}

[1] J. H. Kim, J. H. Park, S. W. Lee, and E. K. P. Chong, "A two layered fuzzy logic controller for systems with deadzones," IEEE Trans. Ind. Electron., vol. 41, pp. 155-162, 1994.

[2] T. Hu and Z. Lin, Control Systems with Actuator Saturation: Analysis and Design. Boston, MA: Birkhäuser, 2001.

[3] C. A. Sacher and G. F. Inbar, "Tracking of the muscle recruitment characteristic during adaptive control of the electrically stimulated knee," Proc. Ann. Int. Conf. IEEE Engineering in Medicine and Biology Soc., vol. 12, no. 5, pp. 2315-2115, 1990.

[4] G. Tao and P. V. Kokotovic, "Adaptive Control of Plants with Unknown Dead-zone,” IEEE Trans. Autom. Control, vol. 39, pp. 59-68, 1994.

[5] K. K. Shyu, W. J. Liu, and K. C. Hsu, "Decentralised variable structure control of uncertain large-scale systems containing a dead-zone," IEEE Proc.-Control Theory Appl., vol. 150, no. 5, pp. 467-475, 2003.

[6] X. S. Wang, C. Y. Su, and H. Hong, "Robust adaptive control of a class of nonlinear systems with unknown dead-zone," Automatica, vol. 40, pp. 407-413, 2004.

[7] A. Isidori, Nonlinear Control System, Springer, New York, 1989.

[8] J. E. Slotine and W. Li, Applied Nonlinear Control, Prentice-Hall, Englewood Cliffs, NJ, 1991.

[9] S. Labiod, M. S. Boucherit, and T. M. Guerra, "Adaptive fuzzy control of a class of MIMO nonlinear systems," Fuzzy Sets and Systems, vol. 151, pp. 59-77, 2005.

[10] S. Tong, H. X. Li, and W. Wang, "Observer-based adaptive fuzzy control for SISO nonlinear systems," Fuzzy Sets and Systems, vol. 148, pp. 355-376, 2004.

[11] J. H. Park, S. J. Seo, and G. T. Park, "Robust adaptive controller for nonlinear system using estimation of bounds for approximation errors," Fuzzy Sets and Systems, vol. 133, pp. 19-36, 2003.

[12] C. H. Wang, H. L. Liu, and T. C. Lin, "Direct Adaptive Fuzzy-Neural Control With State Observer and Supervisory Controller for Unknown Nonlinear Dynamical Systems," IEEE Transactions on Fuzzy Systems, vol. 10, no. 1, pp. 39-49, 2002.

[13] Y. C. Chang, “Adaptive Fuzzy-Based Tracking Control for Nonlinear SISO Systems via VSS and $H^{\infty}$ Approaches," IEEE Transactions on Fuzzy Systems, vol. 9, no. 2, pp. 278-292, 2001

[14] Y. T. Kim and Z. Z. Bien, "Robust adaptive fuzzy control in the presence of external disturbance and approximation error," Fuzzy Sets and Systems, vol. 148, pp. 377-393, 2004.

[15] L. X. Wang, "Stable adaptive fuzzy control of nonlinear systems," IEEE Transactions on Fuzzy Systems, vol. 1, no. 2, pp. 146-155, 1993. 
[16] L. X. Wang, Adaptive Fuzzy Systems and Control: Design and Stability, Prentice-Hill, Englewood Cliffs, NJ, 1994.

[17] J. Wang, A. B. Rad, and P. T. Chan, "Indirect adaptive fuzzy sliding model control: Part I: fuzzy switching," Fuzzy Sets and Systems, vol. 122, pp. 21-30, 2001.

[18] P. T. Chan, A. B. Rad, and J. Wang, "Indirect adaptive fuzzy sliding mode control: Part II: parameter projection and supervisory control," Fuzzy Sets and Systems, vol. 122, pp. 31-43, 2004.

[19] S. Tong and H. X. Li, "Direct adaptive fuzzy output tracking control of nonlinear systems," Fuzzy Sets and Systems, vol. 128, pp. 107-115, 2002.

[20] Y. Tang, N. Zhang, and Y. Li, "Stable fuzzy adaptive control for a class of nonlinear systems," Fuzzy Sets and Systems, vol. 104, pp. 279-288, 1999.

[21] T. P. Zhang, "Stable adaptive fuzzy sliding mode control of interconnected systems," Fuzzy Sets and Systems, vol. 122, pp. 5-19, 2001.

[22] V. I. Utkin, Sliding Modes in Control and Optimization. New York: Springer-Verlag, 1992.

[23] V. I. Utkin, "Variable structure systems with sliding modes,"
IEEE Trans. Autom. Control, vol. AC-22, pp. 212-222, 1977.

[24] J. Y. Hung, W. B. GAO, and J. C. Hung, "Variable structure control: A survey," IEEE Trans. Ind. Electron., vol. 40, pp. 2-22, 1993.

[25] J. J. Slotine and S. S. Sastry, "Tracking control of non-linear systems using sliding surfaces, with application to robot manipulators," Int. J. Control, vol. 38, no. 2, pp. 465-492, 1983.

[26] J. A. Burton and A. S. I. Zinober, "Continuous approximation of variable structure control,” Int. J. Syst. Sci., vol. 17, no. 6, pp. 875-885, 1986.

[27] G. Tao and P. V. Kokotovic, "Adaptive Control of Plants with Unknown Dead-Zones,” IEEE Trans. Autom. Control, vol. 39, no. 1, pp. 59-68, 1994.

[28] E. W. Bai and H. Cho, "Semi-Global Convergence Results on Control of Systems Containing a Dead Zone by an Adaptive Dead Zone Inverse," American Control Conference, 1995. vol. 13 , pp. $2044-2048,1995$

[29] M. Tian, G. Tao, and Y. Ling, “Adaptive Dead-Zone Inverse for Nonlinear Plants," Proceedings of the 35th Conference on Decision and Control, vol.4, pp. 4381-4386, 1996 